\title{
Changes in breast milk lutein concentrations and their associations with dietary lutein intake: a 12-week prospective analytical study
}

\author{
Yaqin Zhang ${ }^{1} \dagger$, Jiao Yang ${ }^{1} \dagger$, Ninghua Huang ${ }^{2}$, Linyan Xiao ${ }^{3}$, Hong Lin ${ }^{4}$, Jiayou Luo $^{4}$, Zhaofeng Zhang ${ }^{1 *}$ and \\ Zhiyong Zou ${ }^{5,6,7 *}$ \\ ${ }^{1}$ Department of Nutrition and Food Hygiene, School of Public Health, Peking University, Beijing 100191, People's Republic of \\ China \\ ${ }^{2}$ Department of Laboratorial Science and Technology, School of Public Health, Peking University, Beijing 100191, People's \\ Republic of China \\ ${ }^{3}$ Liuyang Maternal and Child Care Service Center, Changsha 410300, People's Republic of China \\ ${ }^{4}$ School of Public Health, Central South University, Changsha 410005, People's Republic of China \\ ${ }^{5}$ Institute of Child and Adolescent Health, School of Public Health, Peking University, Beijing 100191, People's Republic of China \\ ${ }^{6}$ Department of Paediatrics, University of Melbourne, Melbourne, VIC 3000, Australia \\ ${ }^{7}$ Key Laboratory of Reproductive Health, National Health Commission of the People's Republic of China, Beijing 100191, People's \\ Republic of China
}

(Submitted 20 March 2019 - Final revision received 2 July 2019 - Accepted 4 July 2019)

\section{Abstract}

We aimed to investigate the trends of breast milk lutein concentrations at different times and their relationship with dietary lutein intake during the 12 weeks after delivery. Breast milk samples were collected from thirty-seven mothers at 4, 8 and 12 weeks postpartum. A HPLC detection method was used to measure breast milk lutein concentrations. Dietary intake was assessed using an FFQ, and then dietary lutein intake was calculated. The correlations between dietary lutein intake and breast milk lutein concentrations during lactation were investigated by Pearson's correlation coefficient. General linear regression models were used to evaluate the optimal regression equation. The mean values of dietary lutein intake at 4,8 and 12 weeks postpartum were 5.22 (sD 3.60), 7.28 (sD 4.30) and 7.33 (sD 4.24) mg/d, respectively. The mean values of breast milk lutein concentrations at 4,8 and 12 weeks postpartum were as follows: 46.41 (sD 41.36), 57.96 (sD 40.00) and 62.33 (sD 30.10) $\mu \mathrm{g} / \mathrm{l}$, respectively. Breast milk lutein concentrations were positively associated with dietary lutein intake at 4 weeks postpartum $(r 0.527, P<0.05)$, which was consistent with the positive correlations observed at 8 and 12 weeks postpartum $(r 0.444, P<0.05 ; r 0.468$, $P<0.05)$ by the sensitivity analysis. Increased dietary lutein intake can increase the concentration of lutein in the breast milk, and women are recommended to increase their dietary intake of green leafy vegetables and fruits that are rich in lutein during the pregnancy and postpartum periods.

Key words: Lutein: Breast milk: Dietary intake: Vegetables and fruits

As one of the major carotenoids in the human retina, lutein exists in high concentrations in the macula lutea and protects against actinic damage and enhances visual function in shortwave dominant-viewing conditions in both infants and adults ${ }^{(1-5)}$. Moreover, present research has reported that lutein plays an important role in the early development and maturation of retinal and visual functions in infants ${ }^{(6,7)}$. However, lutein is not produced by the human body but is present in high concentrations in green leafy vegetables such as spinach and kale ${ }^{(8,9)}$. Because there is a high amount of lutein in vegetables, high lutein intake might reflect an overall healthy diet ${ }^{(10)}$. Moreover, according to the WHO, as the primary and preferred source of nutrition for infants through 6 months of age, breast milk provided infants with all essential nutrients, including lutein ${ }^{(11)}$. Therefore, breast milk lutein concentrations depend principally on the nutritional status of the mother.

Several studies have compared the breast milk lutein concentrations in several countries, and the results showed that the

*Corresponding authors: Z. Zou, fax +86 10 82801178, email harveyzou2002@bjmu.edu.cn; Z. Zhang, fax +86 10 82801178, email zhangzhaofeng@bjmu.edu.cn

$\dagger$ These authors contributed equally to this paper. 
mean values of breast milk lutein concentrations were 25 (sD 19) $\mu \mathrm{g} / \mathrm{l}$ and the concentrations showed notable differences in different regions, ranging from 3 to 44 (SD 18) $\mu \mathrm{g} / \mathrm{l}^{(12,13)}$. In China, Xue et al. ${ }^{(14)}$ reported that the concentration range of lutein in breast milk on $240 \mathrm{~d}$ postpartum was $22-70 \mu \mathrm{g} / \mathrm{l}$ from a cross-sectional study, but there was no cohort study on the changes in breast milk lutein concentrations over time in Chinese women. Furthermore, some studies reported positive correlations between breast milk lutein concentrations and dietary lutein intake in several countries, such as Italy and South Korea ${ }^{(15,16)}$. Compared with the number of studies in foreign countries, there is only one study ${ }^{(17)}$ in China that described the relationship between infant plasma lutein level and the corresponding level in milk; however, the association between dietary lutein intake and the levels of breast milk lutein concentrations in Chinese women has not yet been reported.

Considering the importance of lutein for infants, the aim of the present study was to investigate the changes in breast milk lutein concentrations after 12 weeks of lactation and to explore the association between dietary lutein intake and breast milk lutein concentrations among lactating Chinese women.

\section{Materials and methods \\ Subjects and sample}

We conducted a follow-up study for 12 weeks from January 2017 to September 2018. Lactating women were recruited from a local maternal and child care service centre in Liuyang, Human, China. Liuyang is a county-level city located in eastern Hunan province with a total population of 1.37 million. It is also the largest base of fireworks production and trade base in the world, and its county economy and basic competitiveness ranks 19th of the national top 100 counties. With the help of doctors in Liuyang maternal and child care service centre, we invited breast-feeding mothers to participate with a poster and recruited subjects. The concentrations of lutein in breast milk ranged from 3 to 44 (SD 18) $\mu \mathrm{g} / \mathrm{l}^{(12,13)}$ and $22-70 \mu \mathrm{g} / 1$ in China ${ }^{(14)}$, with a probability of 0.05 and confidence level of $95 \%$; we calculated a reference range for the sample size according to the sample size calculation equation $\left(N=\frac{2\left(z_{\alpha}+z_{\beta}\right)^{2} \sigma^{2}}{d^{2}}\right)$. With references to other studies ${ }^{(14,18)}$ (the range of sample size is 13-540), we determined the sample size ranging from thirty to forty per group. Finally, a total of forty-four lactating women were recruited, and thirty-seven of them finished the follow up with seven lost.

The inclusion criteria were as follows: younger than 35 years old, postpartum BMI $<28 \mathrm{~kg} / \mathrm{m}^{2}$, the baby was born after $37-42$ weeks of gestation, the baby's birth weight was normal ranging from 2500 to $4000 \mathrm{~g}$ and the participant and her baby were in good physical condition with no obvious disease. The exclusion criteria were pre-pregnancy diabetes, fetal anomalies, gestational hypertension, pre-eclampsia, fetal growth restriction, ruptured membranes, postpartum glucose abnormalities, consumption of supplements with lutein, DHA or vitamin E, and insufficient language skills to comprehend the study questionnaires.
All procedures were approved by the Medical Ethics Committee of Peking University. Mothers provided informed consent on their own behalf and on behalf of their infants.

\section{Human milk sample collection}

Lutein is very sensitive to both light and oxygen; therefore, precautions were taken to decrease exposure of breast milk samples to air and/or light throughout all phases of the study. We collected $30 \mathrm{ml}$ milk samples every visit, between 09.00 hours and 10.00 hours from lactating women before maternal breastfeeding on postpartum weeks 4,8 and 12 in a dimly lit room (without direct sunlight exposure) of the Liuyang Maternal and Child Care Service Centre. All samples were collected into polypropylene tubes that were labelled with subject information (including the infant's birth date, date of milk collection and the initials of the mother). The samples were stored at $-80^{\circ} \mathrm{C}$ and then sent to the Peking University Health Science Center until analysis, which was usually performed within $3 \mathrm{~d}$.

\section{Analysis of lutein in human milk}

The determination method of lutein was referred to Khachik classic method and was slightly modified. Validation of characteristics such as specificity, linearity, limit of detection, limit of quantification, accuracy and precision were valued in the previous literature $^{(14,19,20)}$. Frozen human milk was thawed overnight at room temperature, $0.2 \mathrm{ml}$ of internal standard $(2.5 \mathrm{ng} / \mathrm{ml}$ in ethanol), $0.02 \mathrm{~g}$ of butylated hydroxytoluene (BHT), $1 \mathrm{ml}$ of ethanol containing $0 \cdot 1 \% \mathrm{BHT}$ and $20 \mu \mathrm{l}$ of aqueous solution of potassium hydroxide (KOH) (20\%, w/w) were added successively to $1 \mathrm{ml}$ of milk in a $5-\mathrm{ml}$ tube. After mixing, the tube was placed for $30 \mathrm{~min}$ in a shaking water bath at $25^{\circ} \mathrm{C}$ for saponification. Then, $1 \mathrm{ml}$ of a mixed solution of hexane and diethyl ether in a $2: 3(\mathrm{v} / \mathrm{v})$ ratio was added to the samples. After mixing, the samples were centrifuged at $3500 \mathrm{rpm}$ for $3 \mathrm{~min}$; two extractions were performed; the samples were washed with distilled water and dried under $\mathrm{N}_{2}$ at room temperature. The residue was reconstituted in $200 \mu \mathrm{l}$ of dioxane-ethanol (1:1, v/v).

After vortexing for $10 \mathrm{~s}$, the samples were centrifuged at 3000 rpm for $2 \mathrm{~min}$ at $4^{\circ} \mathrm{C}$ and transferred to amber vials. Then, the samples were injected into a HPLC (Agilent 1260 Infinity LC System, Agilent Technologies) and detected at $450 \mathrm{~nm}$. Separation was performed on a Develosil C30 column $(150 \times 4.6 \mathrm{~mm}, 5 \mu \mathrm{m}$ particle size, Nomura Chemical) in series with a Develosil C30 column ( $10 \times 4.0 \mathrm{~mm}, 5 \mu \mathrm{m}$ particle size, Nomura Chemical). A $20 \mu \mathrm{l}$ aliquot of the final extract was injected into the analytical system. The gradient eluting system pumped the mobile phase at a flow rate of $1 \mathrm{ml} / \mathrm{min}$. Mobile phase A was a mixture of acetonitrile-methanol (60:40, v/v), and mobile phase B was a solution of methyl tert-butyl ether. The eluting gradient programme was as follows: 0-15 min, $0 \% \mathrm{~B} ; 15-20 \mathrm{~min}, 42 \% \mathrm{~B} ; 20-30 \mathrm{~min}, 100 \% \mathrm{~B}$. External quantification was performed based on the calibration curve of lutein. The linearity of the calibration curve was verified from the regression coefficient of 0.999. Apart from the internal standard, spiking tests were performed using human milk to ensure adequate recovery of lutein. The average recovery of three replicate analyses was $85 \%$, and the range was $82-88.8 \%$. Repeatability experiments for the same sample provided a relative standard deviation of $1.86 \%$. 
The range of the calibration curve for lutein was $2-50 \mu \mathrm{g} / \mathrm{l}$, the limit of quantification and limit of detection were 0.6 and $5 \mu \mathrm{g} / \mathrm{l}$, respectively.

\section{Dietary lutein intake assessment}

An FFQ was used to assess dietary lutein intake and daily dietary lutein intake was calculated based on dietary lutein intake. Our FFQ for lutein were derived from the Chinese dietary FFQ from the Chinese Centre for Disease Control and Prevention ${ }^{(21,22)}$. It contained thirty-five questions related to the top thirty-one common vegetables and top four common fruits rich in lutein, in each question they were asked the frequency of food per $\mathrm{d} /$ week/month and average amount per serving during the past $30 \mathrm{~d}$.

Up to now, there is no comprehensive database of carotenoid for Chinese foods; therefore, the daily dietary lutein intake was calculated based on the combination of the lutein content in seventy-seven common vegetables and fruits from Chinese study $^{(23,24)}$ and 140 kinds of non-mixed food in Carotenoid Content of U.S. Foods: An Update of the Database (http://ndb. nal.usda.gov/). After excluded foods undetected or low lutein content (less than $50 \mathrm{ug} / 100 \mathrm{~g}$ ) or uncommon in China, finally sixty common vegetables and fruits were listed in the lutein content database for Chinese food (Supplementary Table S1), which was also evaluated in other studies ${ }^{(25,26)}$. Our FFQ covered $58.3 \%$ common food rich in lutein and can estimate majority of daily lutein intake in China because of the striking difference of lutein content in foods (Supplementary Table S1).

\section{Other information collection}

Participants were provided with questionnaires and were asked to answer them while waiting for their check-up at 4, 8 and 12 weeks postpartum. The following demographic and physical characteristic information was collected: age, pregestational BMI, prepartum BMI, gestational weight gain, education level, vision, profession, family monthly income, delivery mode, weight, blood pressure, daily outdoor time and daily sleep time.

The cut-off points for BMI were refer to appropriate body mass index for Asian populations and its implications for policy and intervention strategies ${ }^{(27)}$. We used the suggested categories as follows: less than $18.5 \mathrm{~kg} / \mathrm{m}^{2}$ underweight, $18.5-23 \mathrm{~kg} / \mathrm{m}^{2}$ increasing but acceptable risk, $23-27.5 \mathrm{~kg} / \mathrm{m}^{2}$ increased risk and $27.5 \mathrm{~kg} / \mathrm{m}^{2}$ or higher high risk.

For blood pressure, it was measured using a standardised mercury sphygmomanometer (model XJ1ID) recommended by the National High Blood Pressure Education Program Working Group on High Blood Pressure in Pregnancy ${ }^{(28-30)}$. Blood pressure was measured three consecutive times with an interval of $5 \mathrm{~min}$ from the right arm, after each participant had rested for at least $10 \mathrm{~min}$ and finally a mean of the three measurements was considered as the blood pressure. The cut-offs for normal levels of blood pressure according to Chinese Guidelines for the Prevention and Treatment of Hypertension (revised 2018) $^{(30)}$ : systolic blood pressure is $<120 \mathrm{mmHg}$ and diastolic blood pressure is $<80 \mathrm{mmHg}$.
For vision, experienced eye care professionals measured each eye by using a retro-illuminated logMAR (log minimum angle of resolution) chart with tumbling-E optotypes (Precision Vision) ${ }^{(31)}$; in the present study, we also followed the definitions for population surveys set by the International Council of Ophthalmology in cooperation with the WHO and the International Agency for the Prevention of Blindness ${ }^{(32)}$.

For daily outdoor time, we asked the young mothers how many hours they go out for walk during the past $7 \mathrm{~d}$.

\section{Ethical statement}

All procedures of the present study were approved by the Medical Ethical Committee of the Peking University.

\section{Statistical analysis}

Descriptive data are presented as means and standard deviations for continuous variables and percentages for categorical variables. Changes in lutein content of breast milk over different lactation stages were tested with repeatedmeasures ANOVA. The correlation between breast milk lutein concentrations and dietary lutein intake during lactation was investigated by Pearson's correlation coefficient. Additionally, a sensitivity analysis was completed to verify the correlation. The general linear relationships between dietary lutein intake and breast milk lutein concentrations were estimated with mixed stepwise regression; model 1 was adjusted for age, prepartum BMI and gestational weight gain; model 2 was further adjusted for level of education, daily outdoor time, occupation and family monthly income. Potential associations between breast milk lutein concentrations and dietary lutein intake are presented using standardised $\beta$-coefficients and associated $P$ values. All analyses were carried out using SPSS Statistics Software, version 22.0 (IBM), and a $P$ value of less than 0.5 was considered significant.

\section{Results}

\section{Characteristics of the study participants}

The mean age of the thirty-seven lactating women was 28.9 (sD 4.27) years, and the pregestational BMI of all of the participants was at a normal level. The gestational weight gain was 16.03 (SD 4.27) $\mathrm{kg}$, and the mean blood pressure was at a normal level. The mean outdoor time daily was $1.03(\mathrm{sD} 0.75) \mathrm{h}$, and the mean daily sleep time was 8.23 (sD 2.35$) \mathrm{h}$. The characteristics of the lactating women are summarised in Table 1.

\section{Concentrations of lutein at different times}

Fig. 1 shows the changes in dietary lutein intake and breast milk lutein concentrations. In the present study, the mean values of dietary lutein intake at 4,8 and 12 weeks postpartum were 5.22 (sD 3.60), 7.28 (sD 4.30) and 7.33 (sD 4.24) mg/d, respectively. Dietary lutein intake increased continuously over time and significant changes were observed in dietary lutein intake during the 12 weeks after delivery $(P=0 \cdot 046)$. The mean values of breast milk lutein concentrations at 4,8 and 12 weeks 
Table 1. Characteristics of lactating women at 4 weeks postpartum ( $n 37)$ (Mean values and standard deviations; numbers of participants and percentages)

\begin{tabular}{|c|c|c|}
\hline & Mean & SD \\
\hline Age (years) & 28.92 & 4.27 \\
\hline Pregestational BMl $\left(\mathrm{kg} / \mathrm{m}^{2}\right)$ & 20.59 & $2 \cdot 62$ \\
\hline Prepartum BMI (kg/m²) & $26 \cdot 82$ & 2.06 \\
\hline Gestational weight gain (kg) & $16 \cdot 03$ & $4 \cdot 27$ \\
\hline \multicolumn{3}{|l|}{ College degree or above } \\
\hline$n$ & \multicolumn{2}{|c|}{16} \\
\hline$\%$ & \multicolumn{2}{|c|}{$45 \cdot 7$} \\
\hline \multicolumn{3}{|l|}{ Myopia } \\
\hline$n$ & \multirow{2}{*}{\multicolumn{2}{|c|}{$\begin{array}{c}18 \\
48 \cdot 6\end{array}$}} \\
\hline$\%$ & & \\
\hline \multicolumn{3}{|l|}{ Housewife } \\
\hline$n$ & \multirow{2}{*}{\multicolumn{2}{|c|}{$\begin{array}{c}13 \\
36 \cdot 1\end{array}$}} \\
\hline$\%$ & & \\
\hline \multirow{2}{*}{\multicolumn{3}{|c|}{$\begin{array}{l}\text { Family monthly income } \\
6000 \text { yuan or less }\end{array}$}} \\
\hline 6000 yuan or less & \multirow{2}{*}{\multicolumn{2}{|c|}{4}} \\
\hline$n$ & & \\
\hline$\%$ & \multicolumn{2}{|c|}{$11 \cdot 8$} \\
\hline \multicolumn{3}{|l|}{$6000-10000$ yuan } \\
\hline$n$ & \multirow{2}{*}{\multicolumn{2}{|c|}{$\begin{array}{c}17 \\
50 \cdot 0\end{array}$}} \\
\hline$\%$ & & \\
\hline \multicolumn{3}{|l|}{ More than 10000 yuan } \\
\hline$n$ & \multirow{2}{*}{\multicolumn{2}{|c|}{$\begin{array}{c}13 \\
38 \cdot 2\end{array}$}} \\
\hline$\%$ & & \\
\hline \multicolumn{3}{|l|}{ Caesarean birth } \\
\hline$n$ & \multirow{2}{*}{\multicolumn{2}{|c|}{$\begin{array}{c}8 \\
22 \cdot 2\end{array}$}} \\
\hline$\%$ & & \\
\hline Weight (kg) & 58.83 & 8.23 \\
\hline \multicolumn{3}{|l|}{ Blood pressure } \\
\hline Systolic blood pressure $(\mathrm{mmHg})$ & $109 \cdot 22$ & $7 \cdot 82$ \\
\hline Diastolic blood pressure $(\mathrm{mmHg})$ & $72 \cdot 04$ & 5.54 \\
\hline Daily outdoor time (h) & 1.03 & 0.75 \\
\hline Daily sleep time (h) & 8.23 & $2 \cdot 35$ \\
\hline
\end{tabular}

postpartum were 46.41 (sD 41.36), $57 \cdot 96$ (SD 40.00) and 62.33 (SD $30 \cdot 10) \mu \mathrm{g} / \mathrm{l}$, respectively; the change in breast milk lutein concentrations was similar to that of dietary lutein intake.

\section{Association of dietary lutein intake and breast milk lutein concentrations}

Breast milk lutein concentrations were positively associated with dietary lutein intake at 4 weeks postpartum $(r 0.527, P<0.05)$ (Fig. 2(a)), which was consistent with the positive correlations at 8 and 12 weeks postpartum $(r$ 0.444, $P<0.05 ; r 0.468$, $P<0.05$ ) determined by sensitivity analysis (Fig. 2(b) and (c)).

\section{General linear relationships between dietary lutein intake} and breast milk lutein concentrations

Table 2 shows the general linear model results for the association between dietary lutein intake and breast milk lutein concentrations adjusted for related factors at 4 weeks postpartum. In model 1 , the standardised coefficient for the association was 0.462 (95\% CI 0.197, 0.857; $P=0.003$ ) after adjusting for age, prepartum BMI, gestational weight gain, occupation, family monthly income, and it remained significant $(0 \cdot 382,95 \% \mathrm{CI}$ $0.074,0.761 ; P=0.019)$ after further adjustment for level of education, daily outdoor time, occupation and family monthly income in model 2.

\section{Discussion}

In our study, we found that the longitudinal trends of dietary lutein intake and breast milk lutein concentrations increased during the 12 weeks after delivery in Chinese women. Furthermore, breast milk lutein concentrations were found to be positively associated with dietary lutein intake in Chinese lactating mothers, and the correlation coefficient was obtained.

Up to now, there is no comprehensive database of carotenoid for Chinese foods. In our database (Supplementary Table S1), the contents in spinach (12 $198 \mu \mathrm{g} / 100 \mathrm{~g})$, kale (39550 $\mu \mathrm{g} / 100 \mathrm{~g})$ and parsley (11 434.1 $\mu \mathrm{g} / 100 \mathrm{~g}$ ) are derived from Carotenoid Content of U.S. Foods: An Update of the Database (http://ndb.nal.usda. gov); the content in spinach is consistent with Perry's study (12 $640 \mu \mathrm{g} / 100 \mathrm{~g})^{(8)}$ and higher than a Singapore study $(6051 \mu \mathrm{g} / 100 \mathrm{~g})^{(33)}$, while the content in parsley is higher than Humphries's study $(9924 \mu \mathrm{g} / 100 \mathrm{~g})^{(9)}$. The results also indicated that dietary lutein intake increased over time and was positively associated with breast milk lutein concentrations and dietary lutein intake at 4, 8 and 12 weeks postpartum; these results were consistent with those of previous studies ${ }^{(15,16)}$. In addition, previous research has identified a significant correlation between breast milk lutein concentrations and plasma lutein concentrations ${ }^{(34,35)}$. Regarding the reason dietary lutein intake increased over time in Chinese women, this increase may be influenced by interindividual variability and women's dietary habits ${ }^{(36,37)}$. Liuyang is a county-level city located in eastern Hunan province that is deeply affected by traditional Chinese customs, and all lactating women must experience confinement in childbirth for the first month after delivery $^{(38)}$. Fruits and vegetables were thought to be cold foods and lactating women ate less fruits and vegetables during confinement; therefore, the dietary lutein intake and the breast milk lutein concentrations would be low and would increase over time with the increased intake of fruits and vegetables after confinement ${ }^{(34,35)}$. Based on these studies, dietary recommendations advising a greater intake of fresh fruits and vegetables rich in lutein are extremely useful during pregnancy and lactation.

To date, research on breast milk lutein concentrations in Chinese women has been limited. In our study, the mean breast milk lutein concentration range was $46 \cdot 41-62 \cdot 33 \mu \mathrm{g} / \mathrm{l}$, which was consistent with other studies in China. Lipkie et al. ${ }^{(13)}$ reported the breast milk lutein ranging $22-70 \mu \mathrm{g} / \mathrm{l}$ during 26 weeks postpartum and Xue et $a l .{ }^{(14)}$ found the median concentrations ranging 40-102 $\mu \mathrm{g} / \mathrm{l}$ from 509 healthy mothers in several cities in China. However, the breast milk lutein concentration was a bit higher than that from other countries, such as Northern Ireland $(36.18 \mu \mathrm{g} / \mathrm{l})^{(18)}$, South Korea $(35.00 \mu \mathrm{g} / \mathrm{l})^{(16)}$, Japan (29.07 $\mu \mathrm{g} / \mathrm{l})$, Mexico $(27.25 \mu \mathrm{g} / \mathrm{l})$ and the UK $(12.73 \mu \mathrm{g} / \mathrm{l})^{(39)}$. In addition, most previous studies in other countries reported that breast milk lutein concentrations significantly decreased as the lactation stage progressed, particularly from week 2 to 4 and stabilised at $4-16$ weeks postpartum ${ }^{(13,40,41)}$, and others reported that the concentration reduced over time ${ }^{(18)}$. However, we found that breast milk lutein concentrations increased from 4 weeks to 12 weeks postpartum, which was different from the previous results. This difference may be attributable to differences in several factors, such as distinct dietary 


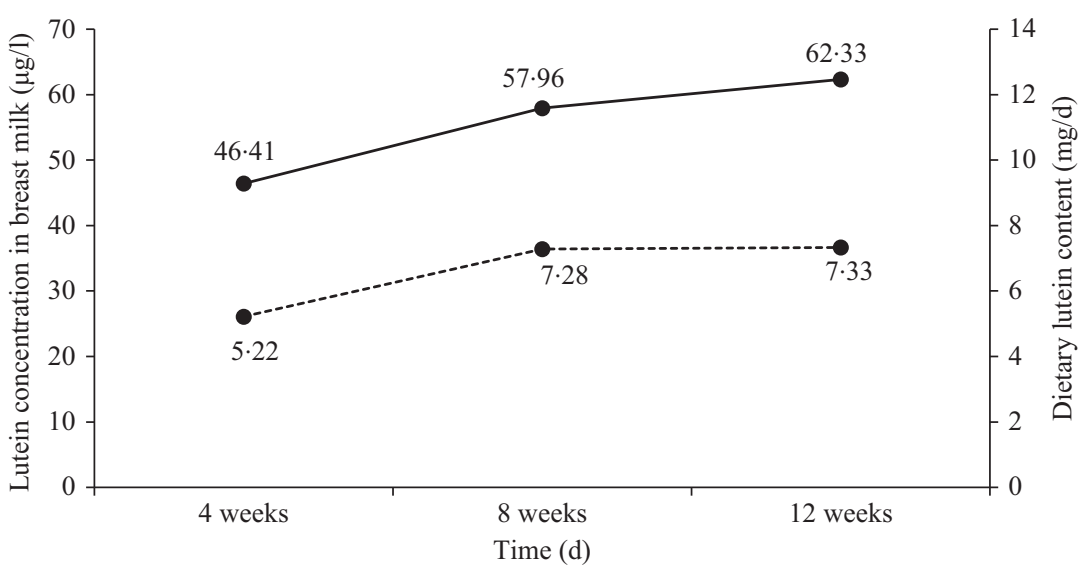

Fig. 1. Changes in dietary lutein content (------) and breast milk lutein concentrations (- - ) over time.
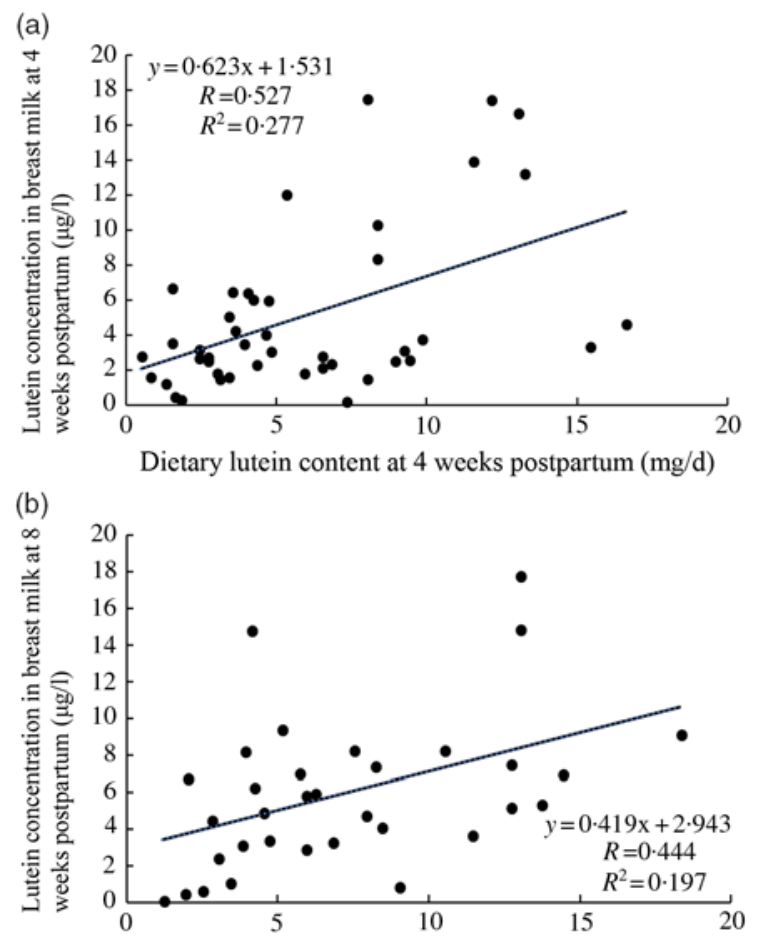

Dietary lutein content at 8 weeks postpartum $(\mathrm{mg} / \mathrm{d})$

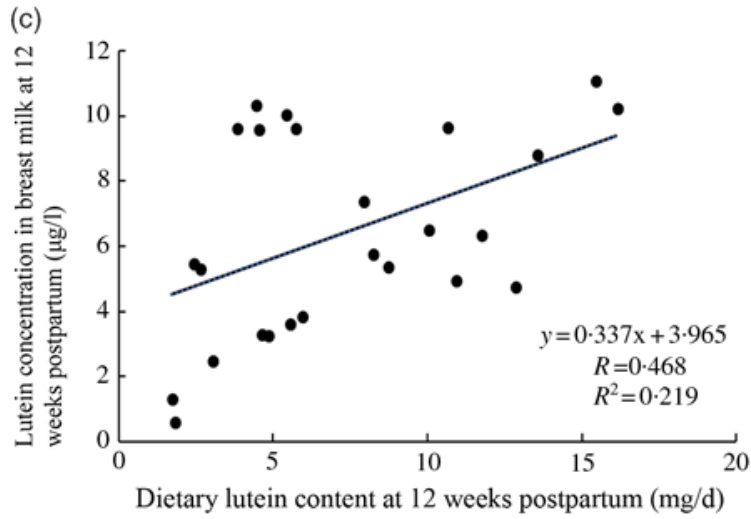

Fig. 2. Association between dietary lutein intake and breast milk lutein concentrations. (a) Association between dietary lutein intake and breast milk lutein concentrations at 4 weeks postpartum; (b) and (c) sensitivity analysis of association of dietary lutein intake and breast milk lutein concentrations. patterns, regional differences ${ }^{(13,39,42)}$, the availability of fruits and vegetables and preferred dish preparation methods. More studies are needed to confirm the regional differences and illuminate their potential mechanisms.

Previous studies have documented that demographic characteristics (age, sex, race/ethnicity, education), BMI and lifestyle factors (physical activity, sunlight exposure, smoking, alcohol consumption) were also reported to be significantly $(P<0.05)$ associated with dietary lutein intake ${ }^{(43-46)}$. In the present study, considering the effect of non-dietary factors on lutein intake, the analyses were adjusted for a substantial number of confounders, including age, prepartum BMI, level of education, occupation, family monthly income, daily outdoor time and gestational weight gain.

The present study has several strengths. Firstly, compared with cross-sectional studies, a longitudinal study is more sensitive to investigate the trends of breast milk lutein concentration during the lactation stage ${ }^{(13)}$. Secondly, an FFQ was used to explore the dietary intake of lactating women in the study. Compared with the 24-h recall method, the FFQ is simple and low cost and it can quickly reveal the types and content of food intake at ordinary times, reflecting the long-term nutrient intake. Moreover, it is more suitable for the evaluation of food and nutrient intake ${ }^{(47,48)}$.

Our findings should also be interpreted in the context of the following limitations. Firstly, breast milk samples at week 0 were not collected in the present study because of the difficulties inherent to the enrolment of postpartum women and budgetary constraints. Secondly, dietary intake data were not available before the participants were pregnant because we started to collect the data after delivery; the dietary lutein intake may be underestimated due to limitations in the food content data, so some daily dietary food intakes might have been ignored. Furthermore, not all foods were covered in our questionnaire; only the top thirty-one common vegetables and top four common fruits rich in lutein were selected. Therefore, though we only covered $58.3 \%$ common food in the database, the results from top thirty-one common vegetables and top four common fruits rich in lutein can estimate majority of daily lutein intake because of the striking difference of lutein content in foods. Meanwhile, we conducted the dietary frequency survey 
Table 2. General linear relationships between dietary lutein intake and breast milk lutein concentrations* (Standardised coefficients and $95 \%$ confidence intervals)

\begin{tabular}{|c|c|c|c|c|c|c|}
\hline \multirow[b]{2}{*}{ Breast milk lutein } & \multicolumn{3}{|c|}{ Model 1† } & \multicolumn{3}{|c|}{ Model $2 \ddagger$} \\
\hline & Standardised coefficient & $95 \% \mathrm{Cl}$ & $P$ & Standardised coefficient & $95 \% \mathrm{Cl}$ & $P$ \\
\hline Constant & -19.391 & $-38.155,-0.627$ & 0.043 & -23.636 & $-46.709,-0.562$ & 0.045 \\
\hline Dietary lutein & 0.462 & $0.197,0.857$ & 0.003 & 0.382 & $0.074,0.761$ & 0.019 \\
\hline
\end{tabular}

* A general linear regression model was used to calculate the association between breast milk lutein concentrations (dependent variable) and dietary lutein intake (independent variable) at 4 weeks postpartum.

$\dagger$ Model 1: adjusted for age, prepartum BMl, and gestational weight gain.

† Model 2: further adjusted for occupation, family monthly income, level of education, and daily outdoor time.

for three times and the results of lutein data were closely associated with the lutein concentration in breast milk, which indicated that the results were relatively consistent.

In conclusion, dietary lutein intake and breast milk lutein concentrations of lactating Chinese women increased with time after delivery and showed a significant positive correlation. Therefore, women of child-bearing age should be encouraged to increase dietary intake of vegetables and fruits that are rich in lutein during pregnancy and the postpartum period.

\section{Acknowledgements}

The authors gratefully acknowledge all the team members and the participating infants and mothers and the great support of Liuyang Maternal and Child Care Service Centre, Changsha.

The present study was funded by the Danone Nutrition Fund (DIC2016-09) and China Scholarship Council.

Z. Z. Y. and Z. Z. F. conceived and designed the project; Z. Y. Q. and Y. J. completed the experiment and prepared the manuscript; X. L. Y., L. H. and L. J. Y. collected the milk samples; Z. Z. Y., Z. Z. F. and L. J. Y. were involved in writing the article and provided a final approval of the submitted and published versions.

None of the authors has any conflicts of interest to declare.

\section{Supplementary material}

For supplementary material/s referred to in this article, please visit https://doi.org/10.1017/S0007114519001727

\section{References}

1. Bernstein PS, Khachik F, Carvalho LS, et al. (2001) Identification and quantitation of carotenoids and their metabolites in the tissues of the human eye. Exp Eye Res 72, 215-223.

2. Beatty S, Koh H, Phil M, et al. (2000) The role of oxidative stress in the pathogenesis of age-related macular degeneration. Surv Ophthalmol 45, 115-134.

3. Stringham JM, Fuld K \& Wenzel AJ (2003) Action spectrum for photophobia. J Opt Soc Am A Opt Image Sci Vis 20, 1852-1858.

4. Stringham JM, Fuld K \& Wenzel AJ (2004) Spatial properties of photophobia. Invest Ophthalmol Visual Sci 45, 3838-3848.

5. Stringham JM \& Hammond BR (2007) The glare hypothesis of macular pigment function. Optom Vis Sci $\mathbf{8 4}, 859$.

6. Hammond BR (2010) Possible role for dietary lutein and zeaxanthin in visual development. Nutr Rev 66, 695-702.
7. Zimmer JP \& Hammond BR (2007) Possible influences of lutein and zeaxanthin on the developing retina. Clin Ophthalmol $\mathbf{1}$, 25-35.

8. Perry A, Rasmussen H \& Johnson EJ (2009) Xanthophyll (lutein, zeaxanthin) content in fruits, vegetables and corn and egg products. J Food Compost Anal 22, 9-15.

9. Humphries JM \& Frederick K (2003) Distribution of lutein, zeaxanthin, and related geometrical isomers in fruit, vegetables, wheat, and pasta products. J Agric Food Chem $\mathbf{5 1}$, 1322-1327.

10. Leermakers ETM, Felix JF, Jaddoe VWV, et al. (2015) Lutein intake at the age of 1 year and cardiometabolic health at the age of 6 years: the Generation R Study. Br J Nutr 114, 970.

11. Bzikowska-Jura A, Czerwonogrodzka-Senczyna A, Oledzka G, et al. (2018) Maternal nutrition and body composition during breastfeeding: association with human milk composition. Nutrients 10, E1379.

12. Canfield LM, Clandinin MT, Davies DP, et al. (2003) Multinational study of major breast milk carotenoids of healthy mothers. Eur J Nutr 42, 133-141.

13. Lipkie TE, Morrow AL, Jouni ZE, et al. (2015) Longitudinal survey of carotenoids in human milk from urban cohorts in China, Mexico, and the USA. PLOS ONE 10, e0127729.

14. Xue Y, Campos-Giménez E, Redeuil KM, et al. (2017) Concentrations of carotenoids and tocopherols in breast milk from urban Chinese mothers and their associations with maternal characteristics: a cross-sectional study. Nutrients $\mathbf{9}$, E1229.

15. Cena H, Castellazzi AM, Pietri A, et al. (2009) Lutein concentration in human milk during early lactation and its relationship with dietary lutein intake. Public Health Nutr 12, 1878-1884.

16. Kim H, Yi H, Jung JA, et al. (2018) Association between lutein intake and lutein concentrations in human milk samples from lactating mothers in South Korea. Eur J Nutr 57, 417-421.

17. Xu X, Zhao X, Berde Y, et al. (2019) Milk and plasma lutein and zeaxanthin concentrations in Chinese breast-feeding motherinfant dyads with healthy maternal fruit and vegetable intake. J Am Coll Nutr, 38, 179-184.

18. Jewell VC, Mayes CB, Tubman TR, et al. (2004) A comparison of lutein and zeaxanthin concentrations in formula and human milk samples from Northern Ireland mothers. Eur J Clin Nutr 58, 90-97.

19. Zou ZY, Xu XR, Huang Y, et al. (2011) High serum level of lutein may be protective against early atherosclerosis: the Beijing atherosclerosis study. Atherosclerosis 219, 789-793.

20. Zou ZY, Xu XR, Lin XM, et al. (2014) Effects of lutein and lycopene on carotid intima-media thickness in Chinese subjects with subclinical atherosclerosis: a randomised, double-blind, placebo-controlled trial. Br J Nutr 111, 474-480.

21. Zhao WH, Huang ZP \& Zhang X (2010) Reproducibility and validity of a Chinese food frequency questionnaire. Biomed Environ Sci 23, 1-38. 
22. Li YP, He YN, Zhai FY, et al. (2006) Comparison of the food intake of the population was evaluated by weighing, retrospective and frequency methods. Chin J Prev Med 40, 273-280.

23. Wang ZX, Dong PC, Sun TT, et al. (2011) Comparison of lutein, zeaxanthin and $\beta$-carotene contents in common foods before and after cooking in Beijing. Chin J Prev Med 45, 64-67.

24. Wang ZX \& Lin XM (2010) Determination and content of lutein, zeaxanthin and beta carotene in common vegetables in Beijing area. Chin J Nutr 32, 290-294.

25. Ma TT (2016) Investigation on lutein intake and absorption metabolism of urban and rural residents in Qingdao. MS Thesis, University of Qingdao.

26. Zhang FD, Zhu YP \& Cai MQ (2012) Correlation analysis between lutein dietary intake and serum content in community of Shanghai. Environ Occup Med 29, 563-565.

27. WHO Expert Consultation (2004) Appropriate body-mass index for Asian populations and its implications for policy and intervention strategies. Lancet 363, 157-163.

28. Chobanian AV, Bakris GL, Black HR, et al. (2003) The seventh report of the Joint National Committee on prevention, detection, evaluation, and treatment of high blood pressure: the JNC 7 report. JAMA 289, 2560-2572.

29. National High Blood Pressure Education Program Working Group on High Blood Pressure in Pregnancy (2000) Report of the National High Blood Pressure Education Program Working Group on high blood pressure in pregnancy. Am J Obstet Gynecol 183, S1-S22.

30. Chinese Committee for the Revision of Hypertension Guidelines (2019) Chinese guidelines for the prevention and treatment of hypertension (revised 2018). Chin Cardiovasc J 24, 24-56.

31. Lougheed T (2014) Myopia: the evidence for environmental factors. Environ Health Perspect 122, A12-A19.

32. Colenbrander A, International Council of Ophthalmology (2002) Visual Standards - Aspects and Ranges of Vision Loss. Sydney: International Council of Ophthalmology.

33. Isabelle M, Lee BL, Lim MT, et al. (2010) Antioxidant activity and profiles of common vegetables in Singapore. Food Chem 120, 993-1003.

34. Sherry CL, Oliver JS, Renzi LM, et al. (2014) Lutein supplementation increases breast milk and plasma lutein concentrations in lactating women and infant plasma concentrations but does not affect other carotenoids. J Nutr 144, 1256-1263.

35. Mackey AD, Albrecht D, Oliver J, et al. (2013) Plasma carotenoid concentrations of infants are increased by feeding a milk-based infant formula supplemented with carotenoids. J Sci Food Agric 93, 1945-1952.
36. Meneses F \& Trugo NMF (2005) Retinol, $\beta$-carotene, and lutein + zeaxanthin in the milk of brazilian nursing women: associations with plasma concentrations and influences of maternal characteristics. Nutr Res 25, 443-451.

37. Tucker KL, Chen H, Vogel S, et al. (1999) Carotenoid intakes, assessed by dietary questionnaire, are associated with plasma carotenoid concentrations in an elderly population. J Nutr $\mathbf{1 2 9}$, 438-445.

38. Raven JH, Chen QY, Tolhurst RJ, et al. (2007) Traditional beliefs and practices in the postpartum period in Fujian Province, China: a qualitative study. BMC Pregnancy Childbirth 7, 8.

39. Jackson JG \& Zimmer JP (2007) Lutein and zeaxanthin in human milk independently and significantly differ among women from Japan, Mexico, and the United Kingdom. Nutr Res 27, 449-453.

40. Song BJ, Jouni ZE \& Ferruzzi MG (2013) Assessment of phytochemical content in human milk during different stages of lactation. Nutrition 29, 195-202.

41. Schweigert FJ, Bathe K, Chen F, et al. (2004) Effect of the stage of lactation in humans on carotenoid levels in milk, blood plasma and plasma lipoprotein fractions. Eur J Nutr 43, 39-44.

42. Jackson JG, Lien EL, White SJ, et al. (1998) Major carotenoids in mature human milk: longitudinal and diurnal patterns. J Nutr Biochem 9, 2-7.

43. Rock CL, Thornquist MD, Neuhouser ML, et al. (2002) Diet and lifestyle correlates of lutein in the blood and diet. J Nutr $\mathbf{1 3 2}$, 525 S.

44. Slattery ML \& Potter JD (2002) Physical activity and colon cancer: confounding or interaction? Med Sci Sports Exerc 34, 913-919.

45. Tormo MJ, Navarro C, Chirlaque MD, et al. (2003) Physical sports activity during leisure time and dietary intake of foods and nutrients in a large Spanish cohort. Int J Sport Nutr Exerc Metab 13, 47-64.

46. Wang Y, Chung SJ, McCullough ML, et al. (2014) Dietary carotenoids are associated with cardiovascular disease risk biomarkers mediated by serum carotenoid concentrations. J Nutr 144, 1067-1074.

47. Crozier SR, Inskip HM, Godfrey KM, et al. (2008) Dietary patterns in pregnant women: a comparison of food-frequency questionnaires and $4 \mathrm{~d}$ prospective diaries. Br J Nutr 99, 869-875.

48. Gabriele N, Dorothee Z, Tilla R, et al. (2007) Long-term reproducibility of a food-frequency questionnaire and dietary changes in the European Prospective Investigation into Cancer and Nutrition (EPIC)-Heidelberg cohort. Br J Nutr $\mathbf{9 8}$, 194-200. 